\title{
Investigation of effect of excitation frequency on electron energy distribution functions in low pressure radio frequency bounded plasmas
}

\author{
Sudeep Bhattacharjee, ${ }^{1,2}$ Trevor Lafleur, ${ }^{1}$ Christine Charles, ${ }^{1}$ and Rod Boswell ${ }^{1}$ \\ ${ }^{1}$ Space plasma, Power and Propulsion, Research School of Physics and Engineering, \\ The Australian National University, Canberra ACT 0200, Australia \\ ${ }^{2}$ Department of Physics, Indian Institute of Technology, Kanpur 208 016, India
}

(Received 26 February 2011; accepted 7 June 2011; published online 5 July 2011)

\begin{abstract}
Particle in cell (PIC) simulations are employed to investigate the effect of excitation frequency $\omega$ on electron energy distribution functions (EEDFs) in a low pressure radio frequency (rf) discharge. The discharge is maintained over a length of $0.10 \mathrm{~m}$, bounded by two infinite parallel plates, with the coherent heating field localized at the center of the discharge over a distance of $0.05 \mathrm{~m}$ and applied perpendicularly along the $y$ and $z$ directions. On varying the excitation frequency $f(=\omega / 2 \pi)$ in the range $0.01-50 \mathrm{MHz}$, it is observed that for $f \leq 5 \mathrm{MHz}$ the EEDF shows a trend toward a convex (Druyvesteyn-like) distribution. For $f>5 \mathrm{MHz}$, the distribution resembles more like a Maxwellian with the familiar break energy visible in most of the distributions. A prominent "hot tail" is observed at $f \geq 20 \mathrm{MHz}$ and the temperature of the tail is seen to decrease with further increase in frequency (e.g., at $30 \mathrm{MHz}$ and $50 \mathrm{MHz}$ ). The mechanism for the generation of the "hot tail" is considered to be due to preferential transit time heating of energetic electrons as a function of $\omega$, in the antenna heating field. There exists an optimum frequency for which high energy electrons are maximally heated. The occurrence of the Druyvesteyn-like distributions at lower $\omega$ may be explained by a balance between the heating of the electrons in the effective electric field and elastic cooling due to electron neutral collision frequency $\nu_{e n}$; the transition being dictated by $\omega \sim 2 \pi \nu_{e n}$. (C) 2011 American Institute of Physics. [doi:10.1063/1.3605021]
\end{abstract}

\section{INTRODUCTION}

Low pressure rf discharges are known to have large degree of spatial nonuniformity and nonequilibrium conditions. ${ }^{1}$ External control parameters such as the discharge boundaries, neutral pressure, and the excitation frequency play a major role in determining the characteristics of low pressure $\mathrm{rf}$ discharges. In the nonlocal regime where the electron energy relaxation length $\lambda_{\epsilon} \gg d$, the discharge length, the plasma electrons are not in equilibrium with the local plasma field. ${ }^{2}$ The excitation frequency can change the dynamics of the discharge considerably by modifying the electron energy distribution function (EEDF). Since a majority of the discharges both for basic research and applications fall under this category where $\lambda_{\epsilon} \gg d$, an investigation of the EEDF with excitation frequency is crucial for an understanding of its role. The present study corresponds to a nonlocal regime since $\lambda_{\epsilon} \sim 3000 \mathrm{~cm} \gg d(10 \mathrm{~cm})$. We investigate the effect of rf excitation that mimics inductive heating as different from capacitively coupled plasmas governed by sheath heating.

With the above viewpoint, recently there has been a lot of investigation of EEDFs in low pressure rf plasmas. EEDFs in helicon discharges operated in the double layer (DL) mode $^{3-5}$ have been looked at. These measurements have been carried out to examine the influence of the double layer on the electrons. Measurements of EEDF both upstream and downstream of the DL have been carried out. Godyak et al. ${ }^{6}$ investigated the EEDFs in inductively coupled argon plasma over a wide pressure and power regimes. In another experiment, the paradoxical behavior of the electron temperature in a low pressure rf discharge was analyzed using measurements of EEDF, ${ }^{2}$ where the combined effects of nonlocality and non-Maxwellian EEDFs have been pointed out. Meige et al. looked at EEDFs in an inductively coupled plasma, investigating the origin of the break energy. However, there are not many studies in low pressure rf discharges where the effect of the excitation frequency of the rf heating field on EEDF is investigated. Most of the studies carried out so far have operated at the standard value of the excitation frequency of $13.56 \mathrm{MHz}$, which is routinely employed in most of the basic research and industrial applications. Some investigations on the effect of $f<13.56 \mathrm{MHz}$ (Ref. 4) have been reported. However, there is a definite lack of investigation over a wide range of excitation frequencies for one to be able to make predictions of the effect of the excitation frequency on EEDFs.

The objective of the present research is to investigate the EEDF in a low pressure rf discharge operated at $1 \mathrm{mTorr}$ in argon, where the excitation frequency $f$ is varied over four decades, 0.01-50 MHz. The investigations are carried out using particle in cell (PIC) simulations. The interplay between the excitation frequency and the electron neutral collision frequency $\nu_{e n}$ is looked at to understand its role in governing the nature of the obtained EEDFs. As electrons transit through the heating region they are locally heated by the antenna electric fields, depending upon the value of $f$, implying that an investigation of the transit time heating 
would be of relevance in predicting their role in electron heating.

The article is organized as follows. In Sec. II, the model used in the PIC simulation scheme is presented. The experimental results from the PIC code are presented in Sec. III. In Sec. IV, the results are discussed. Finally, the results are summarized and conclusions drawn in Sec. V.

\section{MODEL}

In the following, we discuss the PIC simulation scheme. The plasma is confined between two infinite parallel plates separated by $0.1 \mathrm{~m}$. The neutral gas pressure is maintained at $1 \mathrm{mTorr}$ and typical values of the steady state electron density and the plasma space potential are $\sim 2.5 \times 10^{14} \mathrm{~m}^{-3}$ and $\sim 17 \mathrm{~V}$, respectively. The simulation is based upon a well known PIC scheme ${ }^{8-11}$ with Monte Carlo collisions. ${ }^{12}$ The simulation is one dimension (1D) in space $(x)$ and three dimensional (3D) in velocities $(x, y$, and $z$ ). Accurate electron-neutral and ion-neutral collision cross sections are employed to ensure realistic simulations; data can be found in Refs. 13 and 14 for electron-neutral collisions (elastic, exciting, and ionizing), and in Ref. 15 for ion-neutral collisions (elastic and charge exchange). Coulomb collisions are not included in the present study, as their effect on the EEDF would be negligible under the present low density condition.

Until now PIC simulations have been most commonly employed to study capacitive coupling, where the rf voltage is applied to one of the electrodes. ${ }^{1,16}$ Here, the rf heating field is localized at the center of the discharge over a length of $0.05 \mathrm{~m}$. The shape of the rf electric field along the $x$ axis is a top hat and the amplitude is typically $\sim 2.8 \times 10^{4} \mathrm{~V} / \mathrm{m}$. The electrons are heated with a scheme intended to model "inductive excitation," similar to that described by Turner, ${ }^{17}$ but without solving electromagnetic field equations, and has been recently employed by Meige et al. ${ }^{7}$ To simulate the radial electric fields of an actual experiment, the rf electric field is applied in the $y$ and $z$ directions perpendicular to the spatial $x$ dimension where the boundaries are located. This allows us to simultaneously heat electrons in both $y$ and $z$ directions, momentum and energy being transferred to the other directions via electron neutral collisions. The heating current density is maintained at $5 \mathrm{~A} / \mathrm{m}^{2}$. In the present investigation, the heating scheme differs from the previous work by Meige et al., ${ }^{7}$ where the field was applied only along the $y$ direction. We found this leads to nonsymmetric EEDFs in the $y$ and $z$ directions. The amplitude of the rf electric field is taken as uniform over the length of the heating region and localized within it.

The simulations are allowed to run for several thousand rf cycles in order for the system to reach equilibrium. The initial temperatures of the ions and electrons are taken as $0.026 \mathrm{eV}$ (room temperature) and $3 \mathrm{eV}$, respectively. It is found that the typical times required to reach equilibrium vary in the range $\sim 20-30 \mu$ s after the initiation of the discharge. Each simulation particle is a "macroparticle" representing $2 \times 10^{8}$ actual particles. The number of cells along the $x$ axis are 250 and the time step is $\sim 1 \times 10^{-10} \mathrm{~s}$, which allows the simulation to meet the standard stability and accu- racy criteria of the PIC scheme: ${ }^{8-11} \Delta t \ll 0.2 / \omega_{p}$ and $\Delta x \ll$ $\lambda_{D}$, where $\Delta t$ and $\Delta x$ are the spatial and temporal increments used in the simulation, and $\omega_{p}$ and $\lambda_{D}$ are the plasma frequency and the Debye length, respectively. For a typical value of plasma density of $\sim 2.5 \times 10^{14} \mathrm{~m}^{-3}$ and electron temperature $T_{e}$ of $\sim 5 \mathrm{eV}, \lambda_{D} \sim 10^{-3} \mathrm{~m}$ and $\omega_{p} \sim 8.94 \times 10^{8}$ $\mathrm{rad} / \mathrm{s}$.

Depending upon the nature of the obtained EEDFs and the PIC experiments performed here, the results can be grouped in the following frequency regimes: (i) 0.01 $\mathrm{MHz} \leq f \leq 5 \mathrm{MHz}$, (ii) $5 \mathrm{MHz} \leq f \leq 20 \mathrm{MHz}$, and (iii) 20 $\mathrm{MHz} \leq f \leq 50 \mathrm{MHz}$, although the demarcation at the transition frequencies are not rigorous such as to provide distinctly different EEDFs. The EEDFs are presented for three different velocity components $x, y$, and $z$ and mainly at three different locations in the plasma; one near the center $x=0.05$ $\mathrm{m}$, near the edge of the plasma $x=0.012 \mathrm{~m}$ which is closer to the sheath and in the region of the sheath $(x=0.002 \mathrm{~m})$. The EEDFs are measured along the abscissa and the ordinate is in natural $\log$ scale, so that a Maxwellian distribution yields a straight line. For the EEDFs, the electron energies have a resolution of $2 \times 10^{-3} \mathrm{eV}$ and these have been acquired after the system is well into equilibrium (typically $100 \mu \mathrm{s})$ and run for an additional number of time steps $\sim 5 \times 10^{5}$ time steps $(\sim 50 \mu \mathrm{s})$ and averaged. To sum up, the system has a finite size in the $x$ direction $(0.1 \mathrm{~m})$, while it is infinite in the two other directions $y$ and $z$. In Sec. III, the results of the simulations are presented.

\section{RESULTS}

Figures 1(a)-1(c) show typical profiles of the plasma space potential $V_{p}$, the particle densities (electron $N_{e}$ and ion $N_{i}$ ) along the $x$ dimension, and the temporal evolution of the plasma to equilibrium, at an excitation frequency of 20 MHz. From Fig. 1(a), the formation of the sheaths near the boundaries can be seen and the maximum value of the potential at the center is $\sim 17 \mathrm{~V}$. Figure $1(\mathrm{~b})$ shows that the electron and ion densities are $\sim 1.7 \times 10^{14} \mathrm{~m}^{-3}$ at the center of the discharge and decrease uniformly toward the boundaries. The ion densities are higher than the electron densities in the sheath region as expected. The sheath can be approximately taken to begin at a point where the electron and ion densities start to deviate near the boundaries $\sim 0.01 \mathrm{~m}$ from the edge. The sheath thickness is about $10 \lambda_{D}$. Figure 1(c) shows that the discharge attains equilibrium in $\sim 2 \times 10^{5}$ time steps ( $\sim 20 \mu \mathrm{s})$. The discharge was initiated with 1000 simulation particles (electrons and ions) and the equilibrium numbers of particles attained is $\sim 5 \times 10^{4}$. The characteristic features of the bounded plasma and its time evolution to equilibrium is therefore established.

To show the velocity space distribution, typical equilibrium phase space plots of the plasma (electrons and ions) are shown in Figs. 2(a) and 2(b) at an excitation frequency of 20 MHz. The electrons exhibit the expected filling of phase space indicating an uniform distribution of possible velocities, whereas the distribution of the ions are more nonuniform, indicating directed velocities toward the discharge 

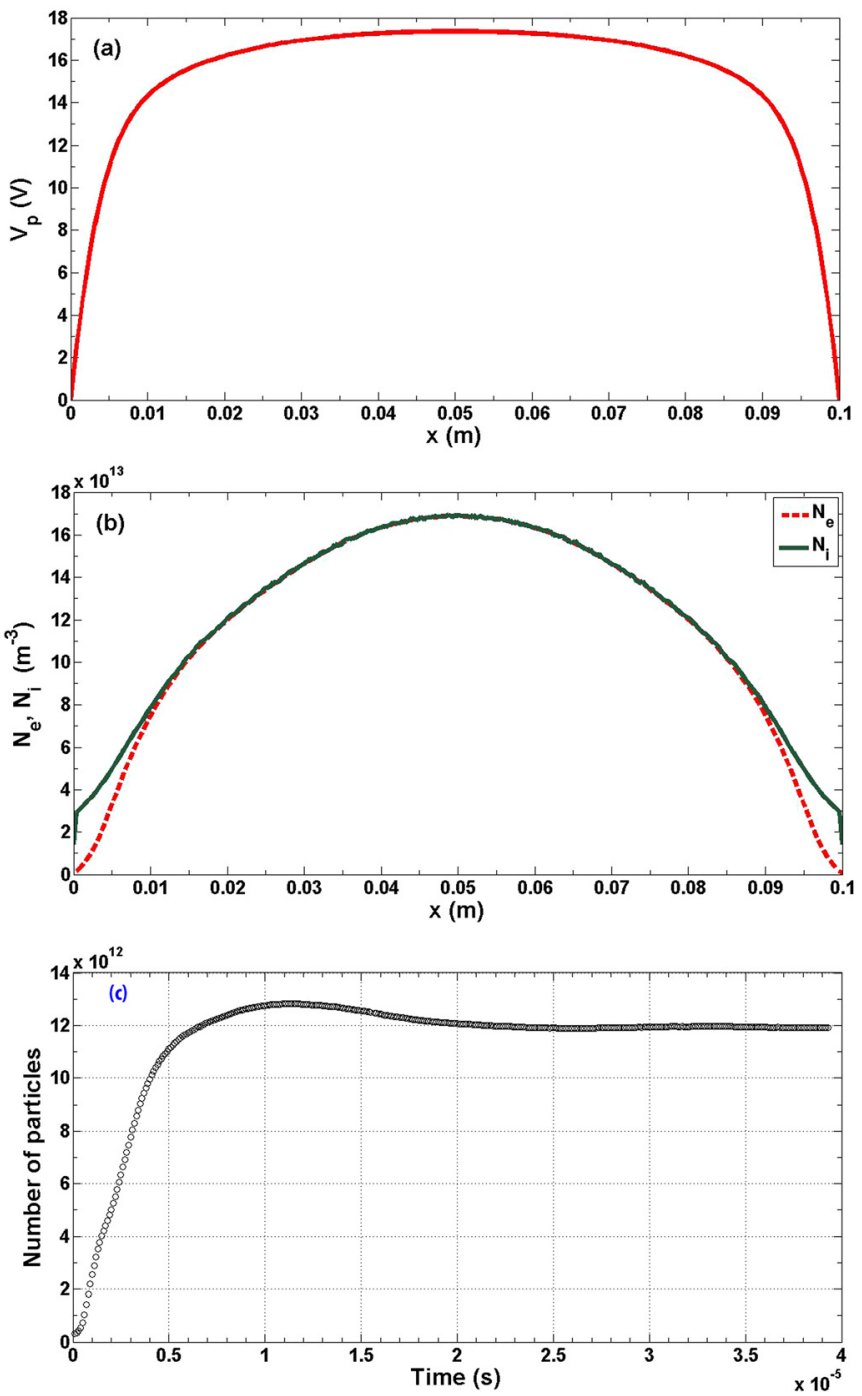

FIG. 1. (Color online) (a) The plasma space potential $V_{p}$, (b) particle densities (electron $N_{e}$ and ion $N_{i}$ ) versus the $x$ dimension of the discharge, and (c) demonstrates the initiation of the discharge to equilibrium which is attained in $\sim 3 \times 10^{5}$ time steps $(30 \mu \mathrm{s})$, for a heating field of frequency 20 MHz. Figure shows the real number of particles.

boundaries as expected, which follow the spatial profile of the electric field.

Figures 3(a)-3(c) show the EEDF in the $x$ direction at an excitation frequency of $5 \mathrm{MHz}, 20 \mathrm{MHz}$, and $50 \mathrm{MHz}$, respectively, at three spatial locations of the discharge $x=0.05 \mathrm{~m}$ (center), $x=0.012 \mathrm{~m}$ (near the edge), and $x=0.002 \mathrm{~m}$ in the sheath region. It is observed that the typical structure of the EEDFs (two Maxwellian with a step) in the $x$ direction is common to all the frequencies even for $f$ $\ll 5 \mathrm{MHz}$ (not shown). The case at $20 \mathrm{MHz}$ (Fig. 3(b)) is described first. The plasma space potential $V_{p}$ at this frequency is shown in Fig. 1(a). It can be seen that as predicted in earlier work, ${ }^{7}$ the break energy of the EEDF shown with dotted lines at the three different spatial locations in the plasma, follow the value of $V_{p}$ at the corresponding spatial location (Fig. 1(a)), $\sim 14 \mathrm{eV}$ at $0.012 \mathrm{~m}, \sim 16.5 \mathrm{eV}$ at $0.05 \mathrm{~m}$, and $3.8 \mathrm{eV}$ at $x=0.002 \mathrm{~m}$ (cf. Fig. 3(b)). This trend agrees well with the results at $5 \mathrm{MHz}$ and $50 \mathrm{MHz}$. It may be noted that outward toward the boundaries $(x=0.012 \mathrm{~m}$ and
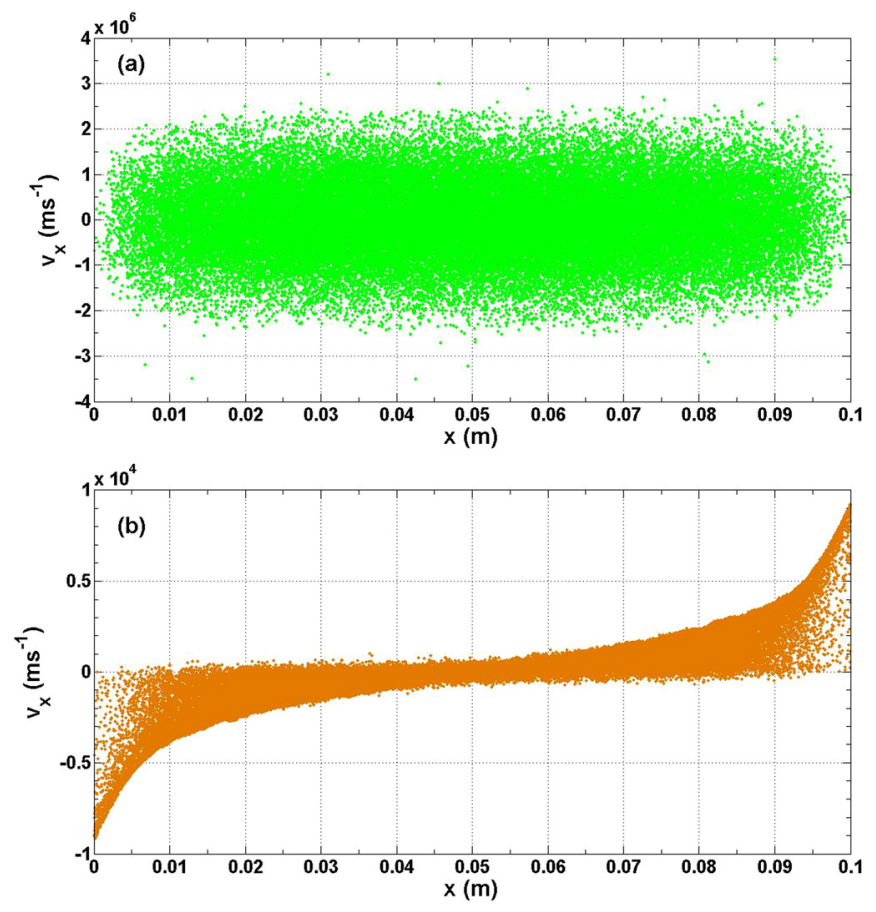

FIG. 2. (Color online) Plasma (a) electron and (b) ion phase space plots at equilibrium $(t=100 \mu \mathrm{s})$ for an excitation frequency of $20 \mathrm{MHz}$.

$0.002 \mathrm{~m}$ ), the EEDFs become deficient in the population of low energy electrons; this is expected because low energy electrons do not have enough energy to overcome the sheath potential barrier. The location $x=0.012 \mathrm{~m}$ is just behind the start of the sheath and does not show as much difference. The characteristic "three temperature structure" of the EEDF has also been reported earlier in some of the EEPFs measured by Godyak et al., ${ }^{6}$ particularly at low pressures $(\leq 10$ mTorr). The temperature of the two populations of electrons $T_{e 1}$ and $T_{e 2}$ above and below the break energy $E_{b}$ has been found and inserted in the figures. It is found that both $T_{e 1}$ and $T_{e 2}$ are higher for $f=20 \mathrm{MHz}$ as compared to the results at 5 $\mathrm{MHz}$ and $50 \mathrm{MHz}$. It is considered that the population of electrons representing the temperature $T_{e 2}$ is a result of electrons scattered to the $x$ direction from the $y$ and $z$ directions. These electrons are unlikely to be observed in most experiments as there are more than one dimension and boundaries. The only chance of observing them would be in highly magnetized and low collision plasmas.

We now investigate the EEDFs in the other two directions ( $y$ and $z$ ). Figures 4(a) and 4(b) show the EEDF in the $y$ and $z$ directions, respectively, for an excitation frequency of $5 \mathrm{MHz}$, taken at the center $(x=0.05 \mathrm{~m})$ and near the edge plasma $(x=0.012 \mathrm{~m})$. It may be noted that with the introduction of the heating field in the two directions $y$ and $z$, the EEDFs have become fairly symmetric in both directions, in contrast to the nonsymmetrical distribution reported by Meige et al., ${ }^{7}$ when the heating field was applied only in the $y$ direction. At $5 \mathrm{MHz}$, the EEDFs tend to become more convex (Druyvesteyn-like). Here, neither the trapped low energy electrons nor the escaping free electrons have distributions which are perfectly Maxwellian. The findings agree reasonably well with Ref. 6 (at 1 mTorr) where with decrease in frequency a similar behavior in the EEPFs was reported. 

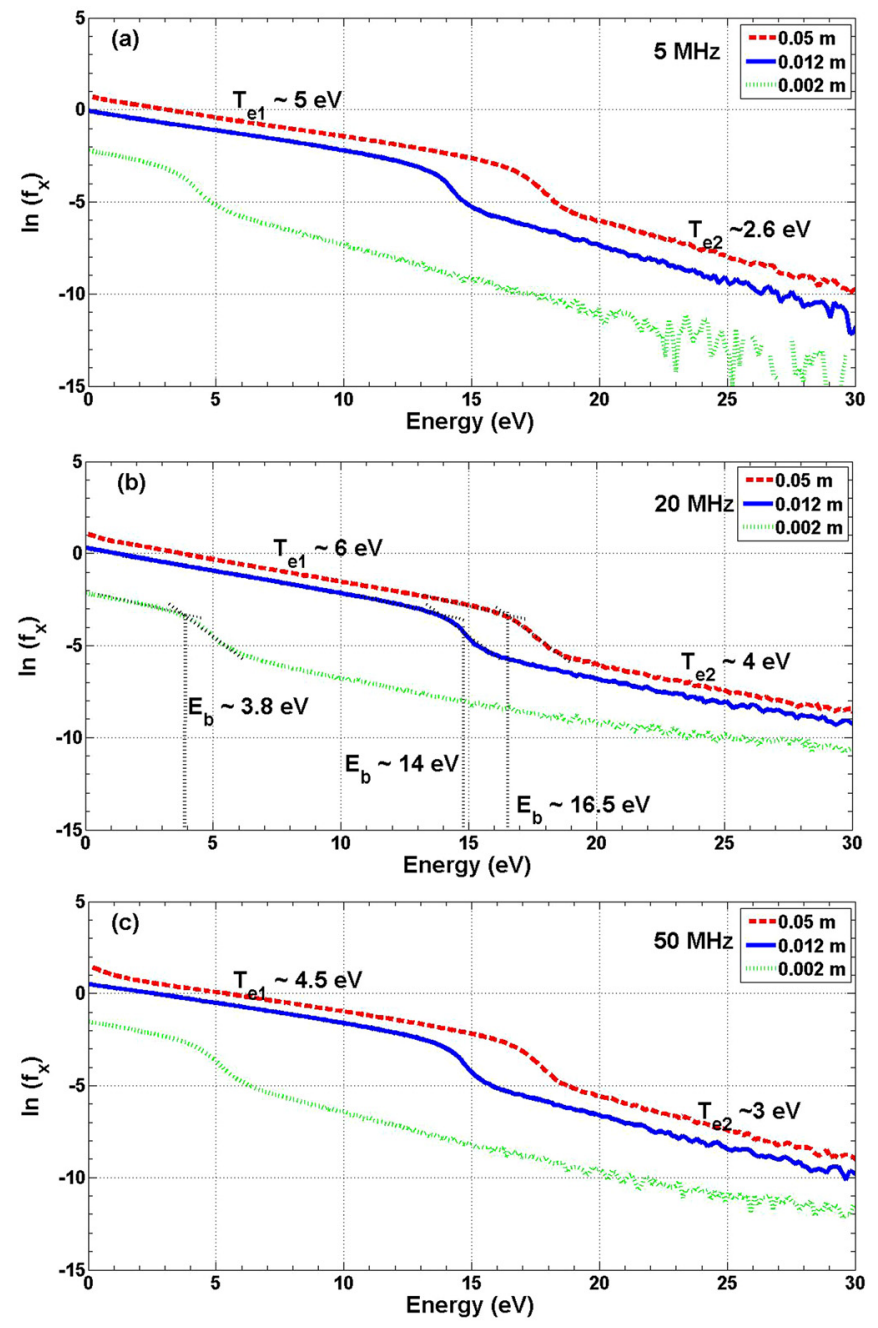

FIG. 3. (Color online) (a) EEDF along the $x$ direction at the center $(x=0.05$ $\mathrm{m})$, near the plasma sheath edge $(x=0.012 \mathrm{~m})$, and in the region of the sheath $(x=0.002 \mathrm{~m})$ of the plasma for a heating frequency of (a) $5 \mathrm{MHz}$, (b) $20 \mathrm{MHz}$, and (c) $50 \mathrm{MHz}$.

However, the maximum excitation frequency employed there was $13.56 \mathrm{MHz}$. In Fig. 4, the break energy is not clearly demarcated but appear to be close to the excitation threshold in argon $(\sim 11.55 \mathrm{eV})$.

Figures 5(a) and 5(b) show the EEDFs in the $y$ direction at a lower $f$ of $0.5 \mathrm{MHz}$ and $1 \mathrm{MHz}$, respectively, at the center $(x=0.05 \mathrm{~m})$ of the plasma. Similar to the result at $5 \mathrm{MHz}$ (Fig. 4) the EEDFs at these low frequencies have a convex profile (Druyvesteyn-like). The break energy $E_{b}$ seems to lie in the range of $10-16 \mathrm{eV}$, i.e., closer to the excitation $(\sim 11.55 \mathrm{eV})$ and ionization thresholds $(\sim 15.76 \mathrm{eV})$, depending on the frequency, e.g., for EEDF at $0.5 \mathrm{MHz}$ it is closer to the excitation threshold and at $1 \mathrm{MHz}$ and $5 \mathrm{MHz}$ it is closer toward the ionization threshold $(\sim 15.76 \mathrm{eV}$ for argon). This is expected as there are no boundaries in the two perpendicular directions and $V_{p}$ should not play a role in dictating the break energy $E_{b}$. We compare the convex type of distributions obtained at lower frequencies $(f \leq 5 \mathrm{MHz})$ with a standard distribution such as a Druyvesteyn distribution which has a convex shape. Such comparisons have been carried out earlier by other authors [cf. Refs. 2, 6, and 18], with an aim to determine the degree of closeness with Druyves-
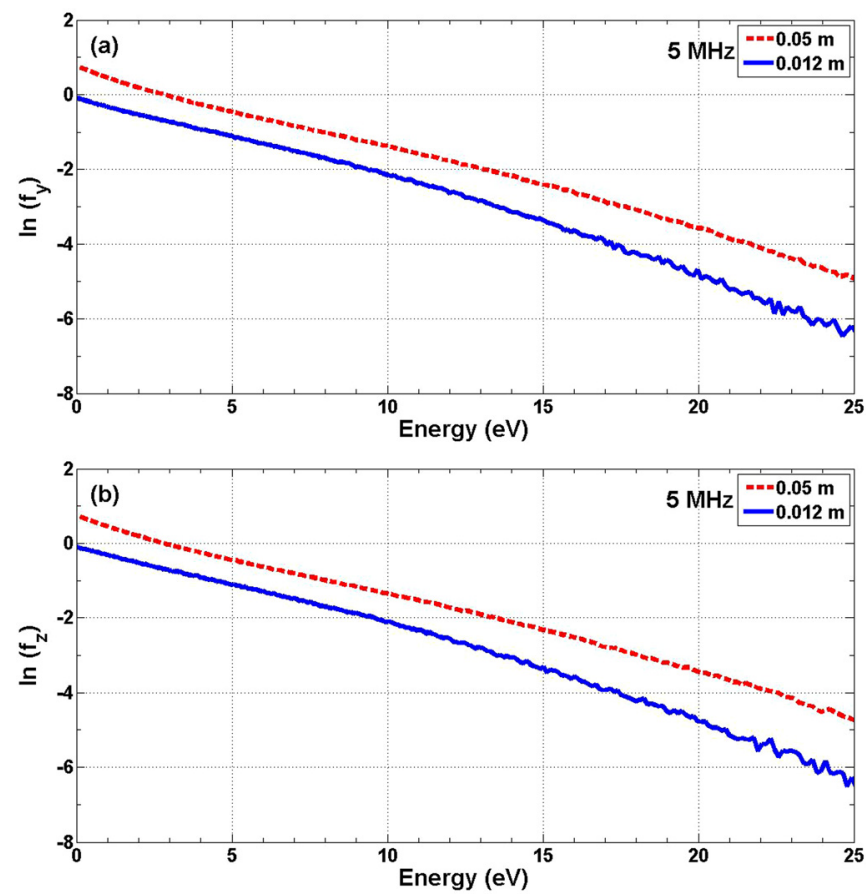

FIG. 4. (Color online) (a) EEDF along the $y$ and (b) $z$ direction at the center $(x=0.05 \mathrm{~m})$ and near the edge $(x=0.012 \mathrm{~m})$ of the plasma for a heating frequency of $5 \mathrm{MHz}$.

teyn distribution from the value of the exponent $r$ in the fitting with $A \exp \left(-E^{r} / B\right)$. (Here, $E$ is the electron energy and $A$ and $B$ are constants). By fitting each EEDF to the function, $A \exp \left(-E^{r} / B\right)$ as shown in Figs. 5(a) and 5(b), the exponential factor $r$ is estimated to be 1.98 for the two cases, ${ }^{18}$ we notice that the depletion of the low energy electrons is less compared to a Druyvesteyn distribution. For a perfectly Druyvesteyn distribution the exponential factor $r=2$ and the population of low energy electrons is more depleted, we note that the fitting is not particularly good near $E=0$. The zero slope at $E=0$ for a Druyvesteyn distribution is difficult to observe even in experiments (e.g., cf. Refs. 6 and 18) and is an ideal situation.

As the excitation frequency is further increased we notice that the EEDFs in the $y$ and $z$ directions transition from a convex type of distribution to a more linear (Maxwellian type) as shown in Figs. 6(a) and 6(b), where the results for $10 \mathrm{MHz}$ and $20 \mathrm{MHz}$ are presented. At $20 \mathrm{MHz}$ (Fig. 6(b)) additionally we observe a "hot tail" in the EEDF, reflecting the manner in which the escaping electrons are heated by the high frequency heating field. It will be discussed later that the development of the "hot tail" is considered to be associated with the transit time heating of the energetic electrons as they pass through the antenna heating field. At frequencies $\geq 10 \mathrm{MHz}$, the break energy is close to the ionization threshold $(\sim 15.76 \mathrm{eV})$. However at $20 \mathrm{MHz}$, we notice that $E_{b}$ is $\sim 25 \mathrm{eV}$ the point where the "hot tail" appears.

Figures 7(a) and 7(b) shows the EEDFs in the $y$ and $z$ directions at a heating frequency of $30 \mathrm{MHz}$ and $50 \mathrm{MHz}$, respectively. The measurements are taken at the center of the discharge $(x=0.05 \mathrm{~m})$. Similar to the results at $10 \mathrm{MHz}$ and $20 \mathrm{MHz}$, the distributions are more Maxwellian with $E_{b}$ 

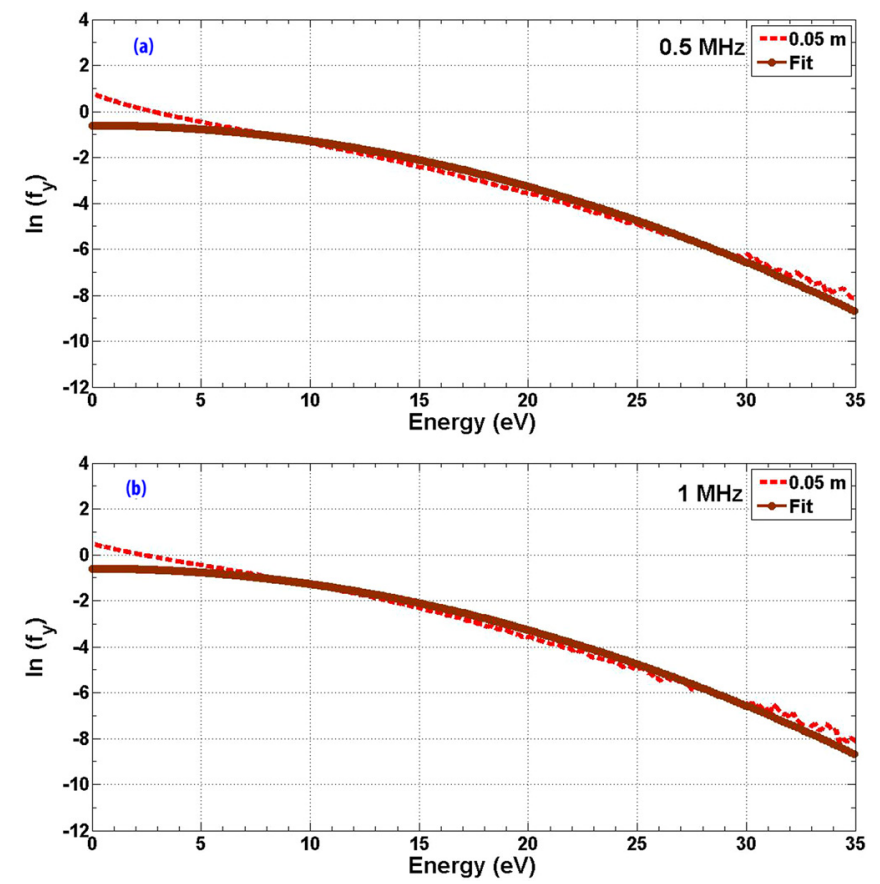

FIG. 5. (Color online) EEDF along the $y$ direction at the center $(x=0.05 \mathrm{~m})$ and near the edge $(x=0.012 \mathrm{~m})$ of the plasma for heating frequency of (a) $0.5 \mathrm{MHz}$ and (b) $1 \mathrm{MHz}$.

closely following the ionization threshold. The effect of the "hot tail" is seen to diminish and is weakly visible at 30 $\mathrm{MHz}$ and almost negligible at $50 \mathrm{MHz}$, indicating that as compared to $20 \mathrm{MHz}$ the heating effect of the high energy electrons diminish at these high frequencies.
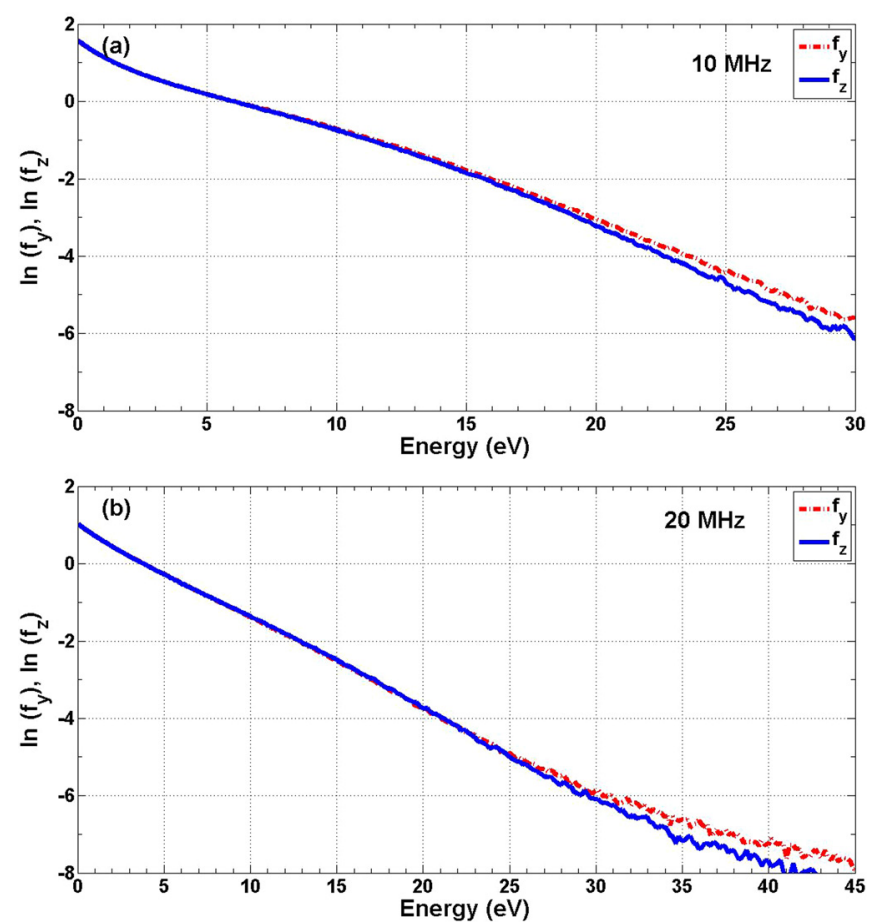

FIG. 6. (Color online) (a) EEDF along the $y$ and $z$ directions at an excitation frequency of (a) $10 \mathrm{MHz}$ and (b) $20 \mathrm{MHz}$, for measurements at the center $(x=0.05 \mathrm{~m})$ of the plasma. Note the occurrence of the "hot tail" at $20 \mathrm{MHz}$.

\section{DISCUSSION}

As seen from the PIC simulations above the EEDFs become convex (Druyvesteyn-like) at frequencies $\leq 5 \mathrm{MHz}$. This may be explained using analytical expression for the effective heating field. The transition frequency closely matches with the operating electron neutral collision frequency $\nu_{e n} \sim 3 \mathrm{MHz}$ at $1 \mathrm{mT}$ Torr pressure and considering an electron temperature of $\sim 5 \mathrm{eV}$. It therefore seems reasonable to investigate the effect of $\nu_{e n}$ on the nature of the obtained EEDFs. As $\nu_{e n}$ or $f$ change relative to each other, the manner in which the electrons are heated would change, which can lead to modification in the shape of the EEDF. The effective heating field as a function of the frequencies can be written as, ${ }^{19,20}$

$$
E_{e f f}=\frac{E_{o}}{\sqrt{\left[1+\frac{\omega^{2}}{\nu_{e n}^{2}}\right]}},
$$

where $E_{o}$ is the amplitude $E=E_{o} \sin \omega t$. The asymptotic values under very low $\left(\nu_{e n} \ll \omega\right)$ and high $\left(\nu_{e n} \gg \omega\right)$ pressure regimes are $E_{\text {eff }} \approx E_{o}\left(\nu_{\text {en }} / \omega\right)$ and $E_{\text {eff }} \approx E_{o}$, respectively. Thus, electron heating can get modified due the effect of both $f$ and $\nu_{e n}$. At $\omega=\nu_{e n}$, we obtain $E_{\text {eff }} \approx E_{o} / \sqrt{2}$. Thus, at high frequencies $\left(\omega \gg \nu_{e n}\right)$ electrons are able to gain energy more efficiently because of the electron-neutral collisions which helps to randomize the directions of the scattered electrons. For the same $E_{o}$, the heating efficiency is decided by a square of the ratio of $\left(\omega / \nu_{e n}\right)$, thus in comparison to phenomena for $f \leq 5 \mathrm{MHz}$, for a higher frequency of $30 \mathrm{MHz}$ one would expect complementary phenomena
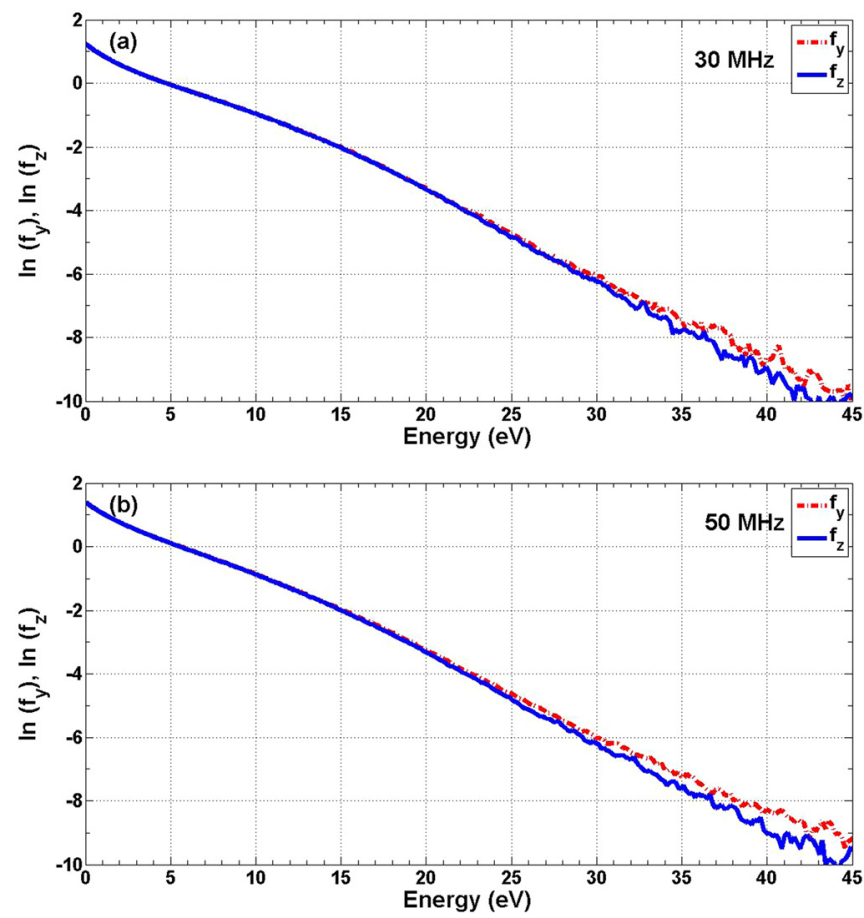

FIG. 7. (Color online) EEDF along the $y$ and $z$ directions at an excitation frequency of (a) $30 \mathrm{MHz}$ and (b) $50 \mathrm{MHz}$, for measurements at the center $(x=0.05 \mathrm{~m})$ of the plasma. 

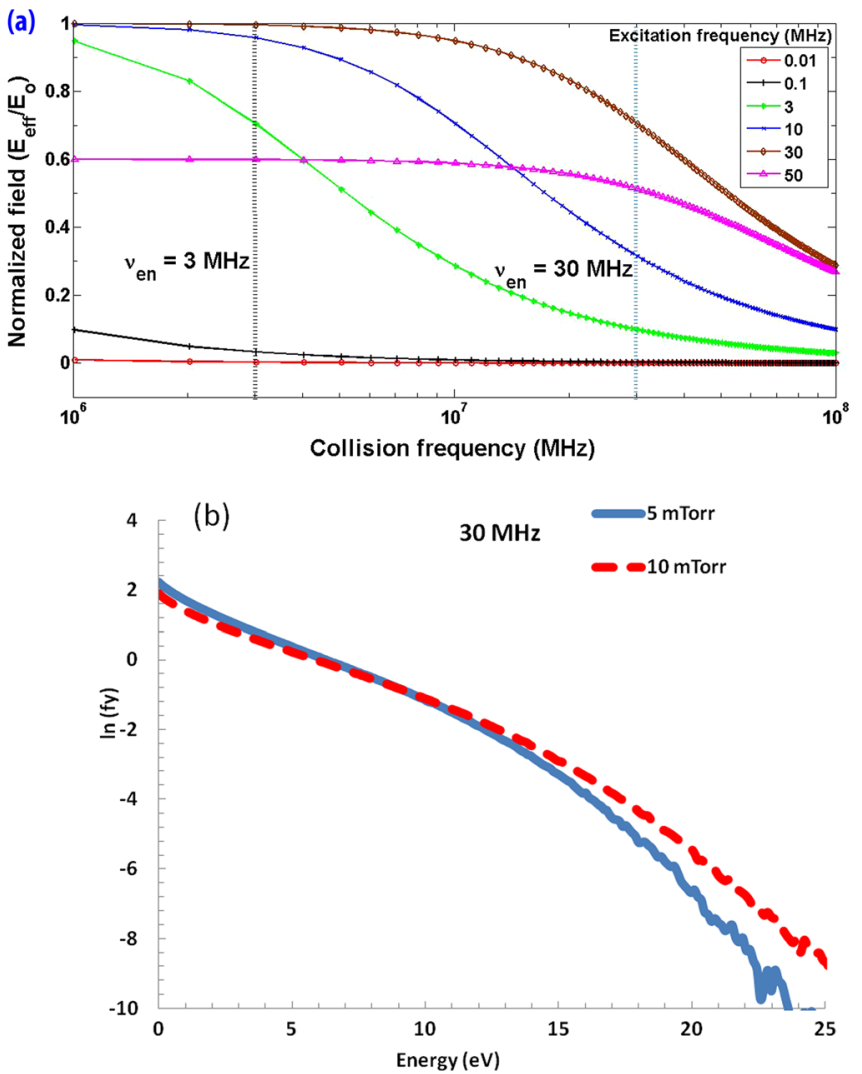

FIG. 8. (Color online) (a) Normalized effective electric field $\left(E_{\text {eff }} / E_{o}\right)$ versus collision frequency $\nu_{e n}$ for a set of excitation frequency. (b) EEDF along the $y$ direction at the center $(x=0.05 \mathrm{~m})$ of the discharge for heating frequency of $30 \mathrm{MHz}$ and collision frequency $\nu_{e n}$ corresponding to a gas pressure of 5 mTorr and 10 mTorr.

(similar distribution functions) at a higher pressure which is 10 mTorr. This is demonstrated in Fig. 8(a) where the normalized effective electric field $\left(E_{\text {eff }} / E_{o}\right)$ is plotted as a function of $\nu_{e n}$ for various values of $f$. The vertical dotted lines indicate $\nu_{e n}$ at $3 \mathrm{MHz}$ and $30 \mathrm{MHz}$. Considering the collision frequency $\nu_{e n}=3 \mathrm{MHz}$ which is the experimental collision frequency (first vertical dotted line), it can be seen that as the excitation frequency $f$ is increased beyond 3 $\mathrm{MHz}$ (e.g., at 10 and $30 \mathrm{MHz}$ ) the normalized effective electric field for a set of collision frequency (and over the whole collision frequency range) increases with $f$ but once again reduces at $f=50 \mathrm{MHz}$. Similarly, the result imply that if we are to operate at a higher $f$ of $30 \mathrm{MHz}$, the normalized $E_{\text {eff }}$ would be comparable to its value at $f=3 \mathrm{MHz}$ (for $\nu_{e n}=3 \mathrm{MHz}$ ) when operated at $\nu_{e n}=30 \mathrm{MHz}$ (which is $p \sim 10 \mathrm{mTorr})$. To appreciate the phenomena the PIC simulations are performed at $30 \mathrm{MHz}$ and at these high pressures (5 mTorr and $10 \mathrm{mTorr})$. Figure 8(b) shows the plot of the effect of $\nu_{e n}(p=5 \mathrm{mTorr}$ and $10 \mathrm{mTorr})$ on the EEDFs at an excitation frequency of $30 \mathrm{MHz}$. From the nature of the EEDFs in the $y$ direction we do see a convex type profile at 5 and $10 \mathrm{~m}$ Torr similar to those obtained at $f \leq 5 \mathrm{MHz}$ and at $1 \mathrm{mTorr}$. It is clear that in order to observe Druyvesteyn-type of distributions at $30 \mathrm{MHz}$, the operating pressure needs to be increased to $\sim 10$ mTorr $\left(\nu_{e n}=30 \mathrm{MHz}\right)$.
In order to get an insight into the mechanism of the "hot tail" observed at $20 \mathrm{MHz}$, we calculated the mean energy $\left\langle E_{i}\right\rangle$ gained by an electron as it transits through one end of the heating region of length $L$ to the other, as a function of the excitation frequency of the rf field. By "hot tail" we mean energetic electrons in the tail region of the distribution function. This is quantified by $\left\langle E_{i}\right\rangle$ and is looked at as a function of the excitation frequency of the rf field. The equation of motion of an electron subjected to a localized field of angular frequency $\omega$ and amplitude $E_{o}$ is

$$
\frac{d v_{y}}{d t}=\frac{q E_{o} \sin \omega t}{m}
$$

where, $q$ and $m$ are the charge and mass of the electron and $v_{y}$ is the $y$ component of the electron velocity at time $t$. The instantaneous velocity of the electron as it transits through the heating region in time $\tau_{i}$ is given by

$$
v_{y}=\frac{q E_{o}}{m \omega}\left[1-\cos \omega \tau_{i}\right] .
$$

The mean energy of the particle during the transit time $\tau_{i}$ is given by

$$
\left\langle E_{i}\right\rangle=\left(\frac{m v_{E}^{2}}{2}\right) \frac{1}{\tau_{i}} \int_{0}^{\tau_{i}}\left[(1-\cos (\omega t)]^{2} d t,\right.
$$

where the transit time $\tau_{i}=L / v_{x}$ and $v_{E}=q E_{o} / m \omega$. At $1 \mathrm{mTorr}$ the transit time is much smaller than the mean collision time. Upon simplification we obtain the normalized heating as
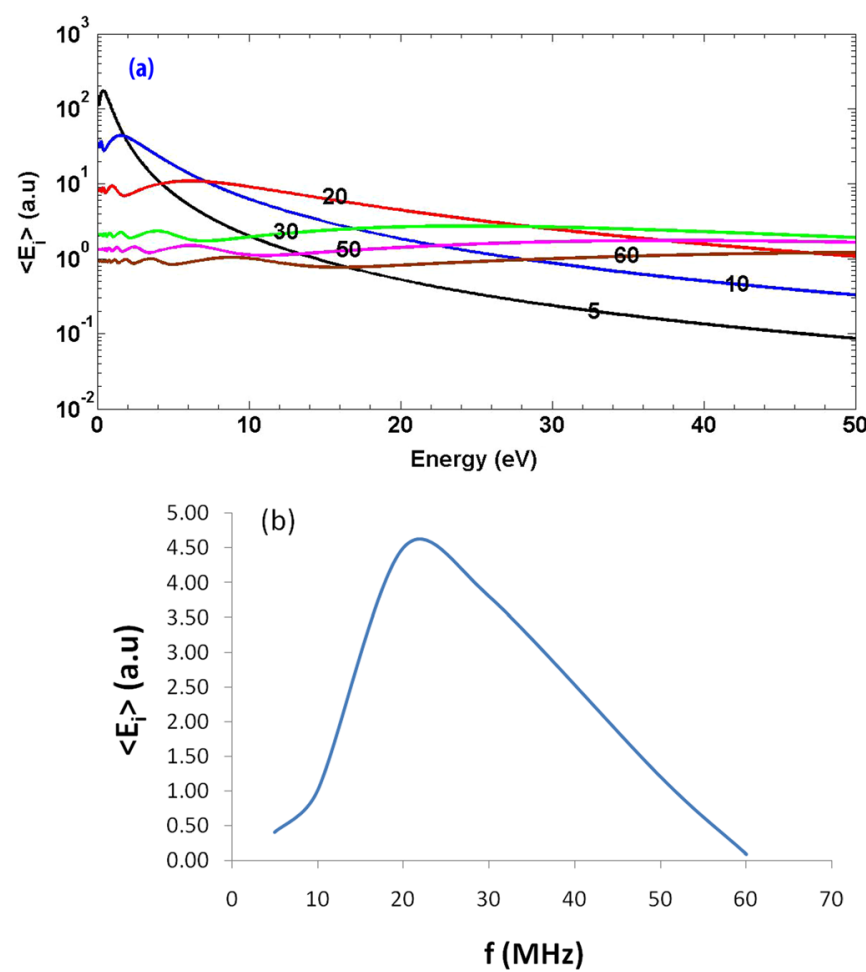

FIG. 9. (Color online) (a) Mean electron energy $\left\langle E_{i}\right\rangle$ gained by the electrons during the transit time $\tau_{i}$ through the antenna heating field region for different excitation frequencies. (b) The mean electron energy $\left\langle E_{i}\right\rangle$ plotted as a function of excitation frequency for electrons with energy of $25 \mathrm{eV}$. 


$$
\frac{\left\langle E_{i}\right\rangle}{\left(m v_{E}^{2} / 2\right)}=\frac{3}{2}-\frac{2 \sin \omega \tau_{i}}{\omega \tau_{i}}+\frac{\sin 2 \omega \tau_{i}}{4 \omega \tau_{i}} .
$$

$\left\langle E_{i}\right\rangle$ given by Eq. (5) is plotted as a function of electron energy in Fig. 9(a). It is noted that depending upon the energy of the electron there is an optimum frequency where the mean energy gained $\left\langle E_{i}\right\rangle$ during its transit time is a maximum. This point is further clarified in Fig. 9(b) where $\left\langle E_{i}\right\rangle$ is plotted as a function of excitation frequency for electrons with energy of $25 \mathrm{eV}$ to clearly demonstrate this effect. Furthermore, it can be noted that at a frequency of $20 \mathrm{MHz}$, the mean energy gained by the high energy electrons with energies $>30 \mathrm{eV}$ are a factor of 10 higher as compared to those at $5 \mathrm{MHz}$.

\section{SUMMARY AND CONCLUSION}

In conclusion, an investigation on the effect of the excitation frequency on EEDFs in a low pressure radio frequency bounded plasma was carried out using PIC simulations that is one dimension (1D) in space $(x)$ and three dimensional (3D) in velocities $(x, y$, and $z$ ). The EEDFs have been found out for electrons with velocities (energies) in all the three directions $x, y$, and $z$ with the excitation frequency varied in the range $0.01-50 \mathrm{MHz}$. Introduction of the heating field in two perpendicular directions ( $y$ and $z$ ) result in symmetric EEDFs in both the directions. Results have indicated that at frequencies $\leq 5 \mathrm{MHz}$ the EEDFs in the $y$ and $\mathrm{z}$ directions have a convex (Dreyvesteyn-like) shape with an exponent of energy, $r=1.98$ which is close to $r=2$ for a true Druyvesteyn distribution. However the depletion of the population at very low electron energies $\leq 5 \mathrm{eV}$ does not seem to match with the predictions of a Druyvesteyn distribution. As the frequency is further increased, for $f \geq 10 \mathrm{MHz}$ the distributions tend to become Maxwellian. For the $x$ directed profiles, almost all the EEDFs have the familiar "two Maxwellian and a step". The temperature of the Maxwellian tail being lower (e.g., Fig. 3(b), $6 \mathrm{eV}$ and $4 \mathrm{eV}$ for $20 \mathrm{MHz}$ ). The EEDFs have been measured at three principal spatial locations in the discharge: at $x=0.05 \mathrm{~m}$ (center), $x=0.012 \mathrm{~m}$ (plasma edge near start of sheath), and $x=0.002 \mathrm{~m}$ (inside the sheath). In regions closer to and in the sheath the populations of the low energy electrons are depleted, which may be understood because low energy electrons do not have enough energy to overcome the potential of the sheath. For the $x$ directed EEDFs the break energy $E_{b}$ is more distinct and closely follows the plasma space potential $V_{p}$, since the electron dynamics for the escaping and trapped electrons is dictated by the boundaries which ultimately sets up $V_{p}$. In the $y$ and $z$ directions where there are no boundaries, $E_{b}$ is not as distinctly visible. However it seems to follow the plasma excitation threshold at $f \leq 0.5 \mathrm{MHz}$ and the ionization threshold for $f \geq 1 \mathrm{MHz}$. At $f=20 \mathrm{MHz}$, a "hot tail" in the EEDF was seen to develop, which seemed to diminish as the frequency was further increased. To understand the convex profiles of the EEDFs and to see how it is affected by the electron neutral collision frequency $\nu_{e n}$, the effective heating field was calculated as a function of $\nu_{e n}$ and $\omega$. The threshold below which the Druyvesteyn type of EEDF appears seem to happen at $\omega=2 \pi \nu_{e n}$. The prediction has been validated with PIC simulations which resulted in convex (Druyvesteyn type) EEDFs at $30 \mathrm{MHz}$ when the pressure was raised to 10 mTorr. The result agrees well with the work of Godyak and Piejak $^{2}$ at $13.56 \mathrm{MHz}$, where the EEDFs were found to resemble Druyvesteyn type distribution when the pressure was raised from 0.03 Torr to 0.3 Torr. The heating of high energy electrons and the generation of a "hot tail" is explained on the basis of transit time heating of electrons as they pass through the heating region of length $L(0.01 \mathrm{~m})$. It is interesting to note that there exists an optimum frequency for which high energy electrons $>20 \mathrm{eV}$ can by preferentially heated to give rise to a "hot tail" as observed in the PIC simulations.

\section{ACKNOWLEDGMENTS}

S. B. gratefully acknowledges support from an Endeavour Research Fellowship of the Australian Government for carrying out this work and the warm hospitality extended by Professor Rod Boswell and Professor Christine Charles during the visit to Australian National University. The short term leave granted by the Physics Department at IIT Kanpur is also gratefully acknowledged which could make this work happen.

${ }^{1}$ M. A. Lieberman and A. J. Lichtenberg, Principles of Plasma Discharges and Materials Processing (John Wiley and Sons, Inc., New Jersey, 2005).

${ }^{2}$ V. A. Godyak and R. B. Piejak, Appl. Phys. Lett. 63, 3137 (1993).

${ }^{3}$ K. Takahashi, C. Charles, R. W. Boswell, T. Kaneko, and R. Hatakeyama, Phys. Plasmas 14, 114503 (2007).

${ }^{4}$ K. Takahashi, C. Charles, R. Boswell, and R. Hatakeyama, Phys. Plasmas 15 (2008).

${ }^{5}$ K. Takahashi, C. Charles, R. Boswell, W. Cox, and R. Hatakeyama, Appl. Phys. Lett. 94, 191503 (2009).

${ }^{6}$ V. A. Godyak, R. B. Piejak, and B. M. Alexandrovich, Plasma Sources Sci. Technol. 11, 525 (2002).

${ }^{7}$ A. Meige and R. W. Boswell, Phys. Plasmas 13, 092104 (2006).

${ }^{8}$ C. K. Birdsall and D. Fuss, J. Comput. Phys. 3, 494 (1969).

${ }^{9}$ A. B. Langdon and C. K. Birdsall, Phys. Fluids 13, 2115 (1970).

${ }^{10} \mathrm{R}$. W. Hockney and J. W. Eastwood, Computer Simulation Using Particles (IOP, Bristol, 1988).

${ }^{11}$ C. K. Birdsall and A. B. Langdon, Plasma Physics Via Computer Simulations (IOP, Bristol, 1991).

${ }^{12}$ V. Vahedi and M. Surendra, Comput. Phys. Commun. 87, 179 (1995).

${ }^{13}$ K. Tachibana, Phys. Rev. A 34, 1007 (1986).

${ }^{14}$ J. L. Pack, R. E. Voshall, A. V. Phelps, and L. E. Kline, J. Appl. Phys. 71, 5363 (1992)

${ }^{15}$ A. V. Phelps, J. Appl. Phys. 76, 747 (1994).

${ }^{16}$ D. Vender and R. W. Boswell, IEEE Trans. Plasma Sci. 18, 725 (1990).

${ }^{17}$ M. M. Turner, Phys. Rev. Lett. 71, 1844, (1993).

${ }^{18}$ M. S. Barnes, J. C. Forster, and J. H. Keller, Appl. Phys. Lett. 62, 2622 (1993).

${ }^{19}$ W. P. Allis, in Handbuch der Physik, edited by S. Flugge (Springer-Verlag, Berlin, 1956), Vol. 21, p. 283.

${ }^{20}$ R. B. Piejak, V. A. Godyak, and B. M. Alexandrovich, Plasma Sources Sci. Technol. 1, 179 (1992). 